Check for updates

Cite this: RSC Adv., 2017, 7, 55935

Received 25th October 2017

Accepted 6th December 2017

DOI: 10.1039/c7ra11792b

rsc.li/rsc-advances

\section{Highly luminescent silver nanocluster-doped fluorophosphate glasses for microfabrication of 3D waveguides}

\author{
H. Fares, (D) ${ }^{* a}$ S. N. C. Santos, ${ }^{b}$ M. V. Santos, ${ }^{b}$ D. F. Franco, ${ }^{a}$ A. E. Souza, ${ }^{a}$ D. Manzani, ${ }^{c}$ \\ C. R. Mendonça ${ }^{b}$ and M. Nalin ${ }^{a}$
}

Highly photoluminescent glasses were prepared by embedding silver nanoclusters (Ag NCs) in a fluorophosphate matrix using a melt-quenching method. The NCs sizes were varied through silver concentration and thermal annealing. Depending on the NC size and excitation wavelength, different colors can be generated ranging from blue to yellow. The tunable photoluminescence and the excellent stability of Ag NCs even after heat treatment are attractive features for photonic applications. In this sense, we demonstrated the fabrication of luminescent tridimensional waveguides in as-prepared glasses by femtosecond laser micromachining. The waveguides were found to emit and guide white light when excited at $325 \mathrm{~nm}$. We foresee that this study presents promising results for the development of new photonic devices and data storage.

\section{Introduction}

Glasses are of great importance on the development of photonic devices and optical components owing to their relatively easy manipulation and their versatility to be obtained in different shapes and sizes. ${ }^{1}$ In addition, they provide many unique characteristics, including excellent transparency, low cost, high optical damage threshold, and last but not least, the easiness of doping with active ions. ${ }^{2}$ Numerous applications of active glasses have been proposed for new technologies in optics and photonics such as fiber optics, fiber amplifiers, solid-state lighting, solar cells technology, light emitting diodes (LEDs) and other photonic devices. ${ }^{3-7}$ Among these numerous applications, significant attention has been drawn recently on the microfabrication of three-dimensional (3D) waveguides for femtosecond laser direct writing (FLDW).$^{8-10}$ FLDW has been explored as an attractive processing tool for spatially controlled structural modification in transparent materials. ${ }^{11}$ The irradiation with tightly focused femtosecond laser pulses inside materials, makes 3D microfabrication possible due to the confinement of the nonlinear interactions within the focal volume. In addition, as a consequence of the nonlinear interaction during micromachining, the optical properties can be locally changed, resulting in changes in the refractive index.

${ }^{a}$ Institute of Chemistry, São Paulo State University, UNESP, Araraquara, SP, Brazil. E-mail: fares.hssen@gmail.com

${ }^{b}$ São Carlos Institute of Physics, University of São Paulo, IFSC-USP, São Carlos, SP, Brazil

'Institute of Chemistry of São Carlos, University of São Paulo, 13566-590, São Carlos, SP, Brazil
Depending on the experimental conditions, fs-laser micromachining can lead to waveguides with the refractive index changing from $10^{-4}$ to $10^{-2}$, allowing propagation of fundamental or higher-order modes. ${ }^{12}$ Recent research on the microfabrication of waveguides focused on glasses doped with $\mathrm{Ag}$ NCs due to their superior photosensitivity to the high repetition rate of the femtosecond laser. ${ }^{9}$ The combination of their tunable photoluminescence properties with the high photosensitivity could open the opportunities for the fabrication of different photonic devices, including 3D data storage and luminescent waveguides.

In this direction, we demonstrate for the first time the feasibility of Ag NCs doped fluorophosphate glasses for the realization of luminescent $3 \mathrm{D}$ waveguides. The mechanism of formation of the metallic particles within the glass and the influence of the particle size on the emission properties of $\mathrm{Ag}$ NCs were also systematically investigated.

\section{Experimental details}

\subsection{Glass synthesis and characterization}

Fluorophosphate glasses with chemical composition $(50-x)$ $\mathrm{NaPO}_{3}-20 \mathrm{PbF}_{2}-20 \mathrm{BaF}_{2}-10 \mathrm{YF}_{3}-x \mathrm{AgNO}_{3}(\mathrm{~mol} \%)$ were prepared by conventional melt-quenching method using high purity commercial materials (99.9\%, Sigma-Aldrich). Silver nitrate $\left(\mathrm{AgNO}_{3}\right)$ was added in different molar concentrations with $x=0,1,2,3$, and $5 \mathrm{~mol} \%$ and labeled as Ag0, Ag1, Ag2, Ag3 and Ag5, respectively. A Pt/Au crucible containing approximately $10 \mathrm{~g}$ of mixed batches was heated at $1000{ }^{\circ} \mathrm{C}$ for $1 \mathrm{~h}$ under air atmosphere. Afterward, the melts were casted into a stainless steel mold preheated near to the glass transition temperature 
$\left(T_{\mathrm{g}}\right)$, at $350{ }^{\circ} \mathrm{C}$. To avoid the accumulated mechanical constraints, the obtained samples were left at such temperature during $3 \mathrm{~h}$ before being cooled down to room temperature. Further, the sample Ag5 was cut and optically polished with a thickness of $2 \mathrm{~mm}$, and each piece was heat-treated at $400{ }^{\circ} \mathrm{C}$ (slightly above the glass transition temperature) for periods of 2 , 4 and $6 \mathrm{~h}$. At this temperature, the glass viscosity decreases, allowing ion diffusion into the glass structure and providing aggregation and growth of silver NCs. The heat-treated samples are denoted as $R y$, where $y$ represents the annealing time (in hours) at $400{ }^{\circ} \mathrm{C}$.

Differential scanning calorimetry (DSC) measurements for prepared glasses were carried out at temperatures ranging from 200 to $800{ }^{\circ} \mathrm{C}$ using a Netzsch DSC Pegasus 404F3. In such conditions, The DSC scans were recorded using $10 \mathrm{mg}$ as-cast glass specimens, which were powdered and heated in a platinum crucible under a flowing nitrogen atmosphere. The measurements were performed at a scan rate of $10{ }^{\circ} \mathrm{C} \mathrm{min}{ }^{-1}$ for heating and cooling.

Transmission electron microscopy (TEM) investigations were carried out using a Philips CM200 operating at $200 \mathrm{kV}$, equipped with X-ray energy dispersive spectroscopy (EDS) Bruker model XFlash 6TI30. UV-vis absorption spectra and transmission spectra were recorded using a Varian Cary 500 double beam spectrophotometer. Excitation and emission spectra were obtained using a Fluorolog (Horiba Jobin Yvon) fluorimeter equipped with a double monochromator and a photomultiplier tube sensitive from 250 to $825 \mathrm{~nm}$. Slits were adjusted to lead to a resolution of $1 \mathrm{~nm}$ for both excitation and emission. All measurements were obtained from glass pieces at room temperature and corrected by the instrument response.

\subsection{Microfabrication of optical waveguides}

Waveguides were fabricated inside the glass bulk sample by femtosecond laser micromachining using 50 fs pulses from a Ti:Sapphire laser oscillator operating at $800 \mathrm{~nm}$ with a repetition rate of $5 \mathrm{MHz}$. The laser pulses with $32 \mathrm{~nJ}$ were focused through a microscope objective (NA $=0.65)$ inside the glass volume, while the sample was freely moved with a speed of
$90 \mu \mathrm{m} \mathrm{s}^{-1}$ perpendicularly to the laser beam using a $x y z$ translational stage. Two different light-coupling systems based on He-Ne (632.8 nm) and He-Cd lasers were used to characterize the guiding properties of the fabricated waveguide. In both coupling systems, a $20 \times$ objective lens was used to couple the laser and a CCD camera was used to observe the propagation mode. Total losses were determined by measuring the input and output power on the waveguide at $632.8 \mathrm{~nm}$. The spectrum of guiding light was obtained using a fiber-based spectrometer. Details about our experimental setup for laser writing and optical coupling system are available in ref. 10 .

\section{Results and discussion}

\subsection{Optical properties and nanostructure analysis}

Fig. 1 shows the photography of prepared glass samples under daylight (Fig. 1(a)) and UV light (Fig. 1(b)). One can see that the as-prepared glasses doped with Ag NCs are homogeneous, free of strains and presenting yellowish color for samples containing high concentration of $\mathrm{AgNO}_{3}$. Under UV excitation, the undoped sample does not show any appreciable luminescence, while the doped ones result in a yellow emission, increasing in intensity according to $\mathrm{Ag}$ concentration.

Fig. 2(a) reveals the transmission spectra of the as-prepared glasses. The glasses show a good transmittance $(>80 \%)$ which decrease at higher silver concentration. In addition, a weak absorption band located at about $430 \mathrm{~nm}$ is detected in all glass samples except for the undoped glass, as shown in Fig. 2(b). This band is attributed to the surface plasmon resonance (SPR) absorption of $\mathrm{Ag}$ NCs consisted of a tiny number of $\mathrm{Ag} .{ }^{13} \mathrm{In}$ addition, the SPR band becomes more intense as a function of $\mathrm{AgNO}_{3}$ concentration, indicating an increase on the $\mathrm{Ag}$ NCs size. The evolution of $\mathrm{Ag}$ species under different doping conditions can also be viewed from the pictures in the inset of Fig. 2 .

In order to study the evolution of silver NCs sizes as a function of $\mathrm{Ag}$ doping, transmission electron microscopy (TEM) analysis were carried out for samples Ag2 and Ag5. TEM images, selected area electron diffraction pattern (SAED) and the particle diameter distribution are shown in Fig. 3 for Ag2 sample, and in Fig. 4 for Ag5 sample. The size distributions of

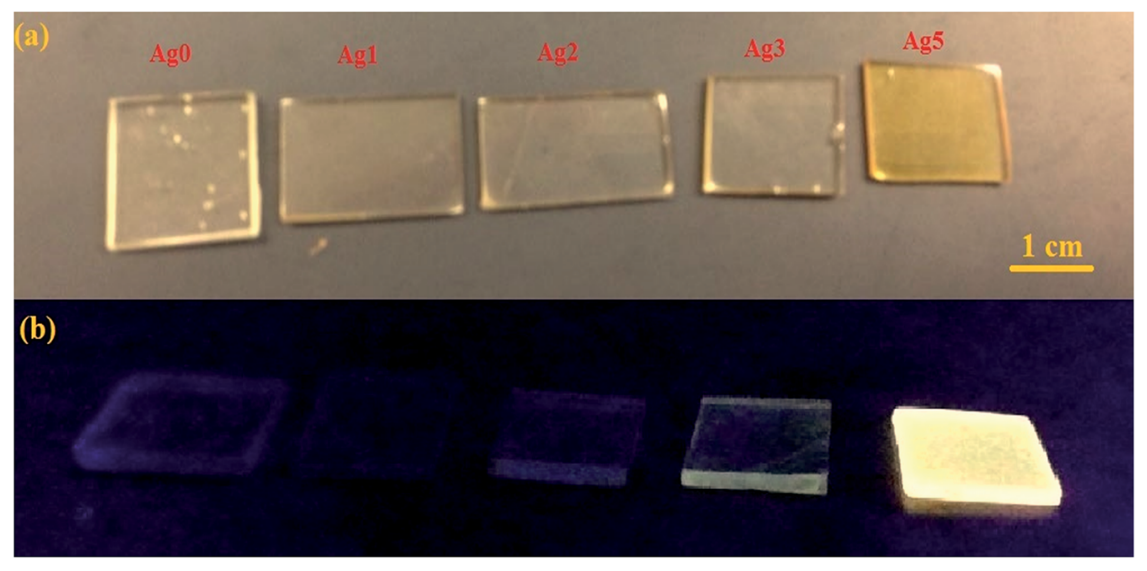

Fig. 1 (a) Daylight pictures, (b) luminescence images excited by a UV lamp at $365 \mathrm{~nm}$ for all prepared glasses. 

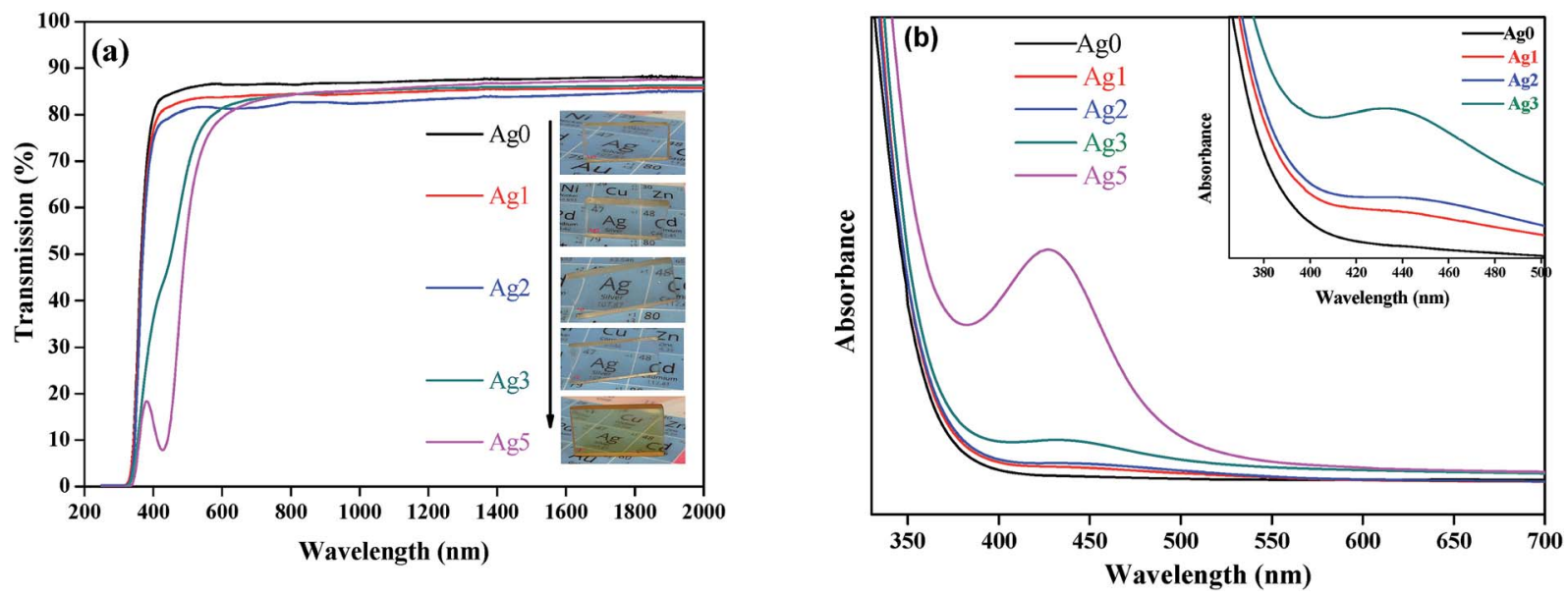

Fig. 2 (a) Transmission spectra of glass samples as a function of $\mathrm{AgNO}_{3}$ concentration with their corresponding photograph presented in inset. (b) Absorption spectra of Ag NCs doped prepared glasses.
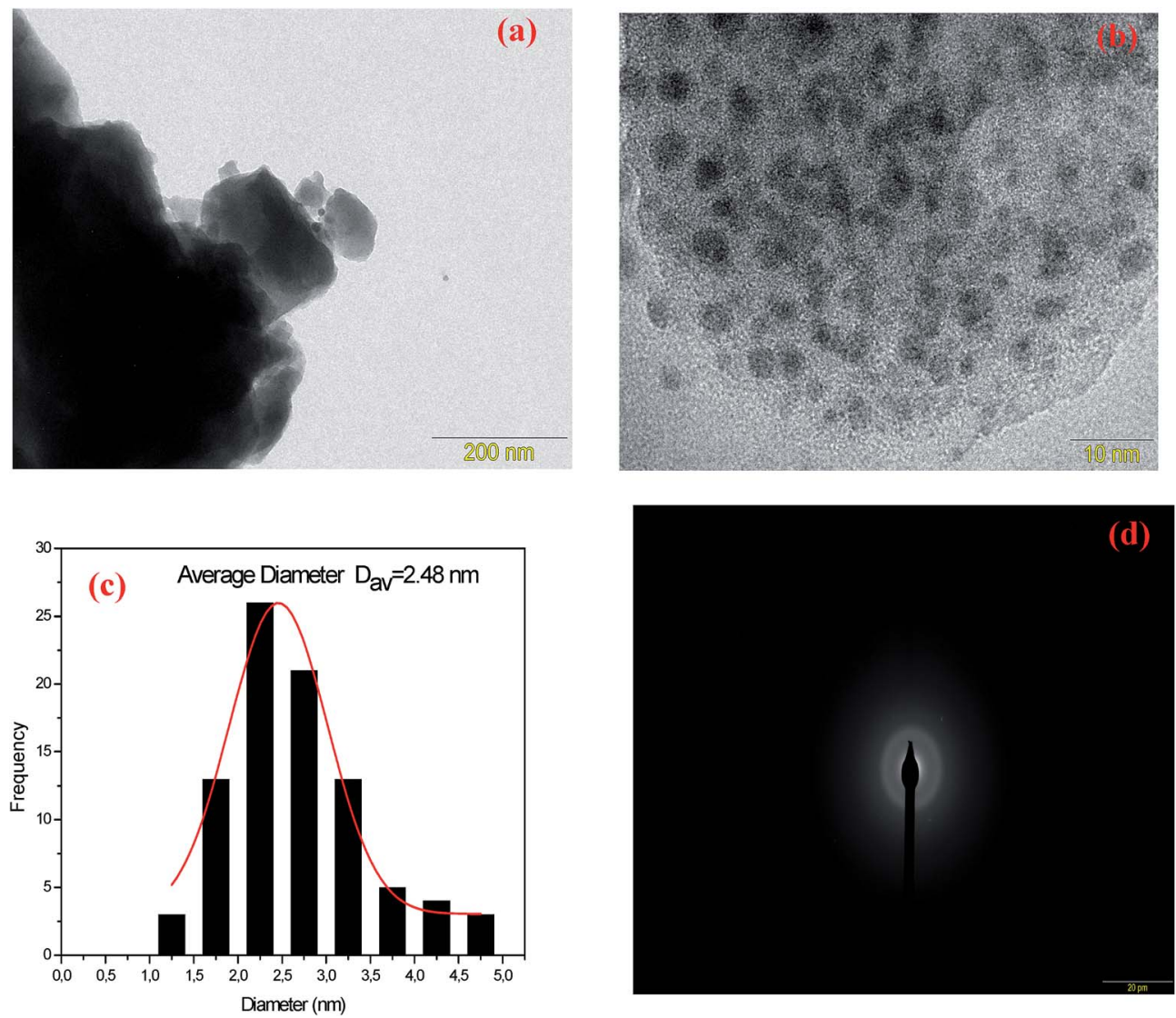

Fig. 3 (a) TEM images (b) at $10 \mathrm{~nm}$ scale, (c) particle size distribution curve and (d) selected area electron diffraction pattern (SAED) of Ag3 glass samples.

Ag NCs of Ag2 and Ag5 samples were obtained by the analysis of 200 to 250 particles. As shown in TEM images (Fig. 3 and 4), the metallic Ag species are visible as dark spots distributed across the glass matrix. Additionally, the amorphous character of Ag NCs is evidenced by SAED pattern (Fig. 3(d) and 4(d)), which contains only diffuse diffraction rings typical for an amorphous phase. More importantly, the quantitative analysis of the size distribution histograms reveals that the average size does increase with the $\mathrm{Ag}$ doping. In particular, the number of NCs with diameter higher than $3 \mathrm{~nm}$ increases with the increase of $\mathrm{AgNO}_{3}$ concentration.

It is worthy to say that the formation and growth of Ag NCs occur without any heat treatment, and can be described by a three-step mechanism as follow: (i) the procedure starts with 

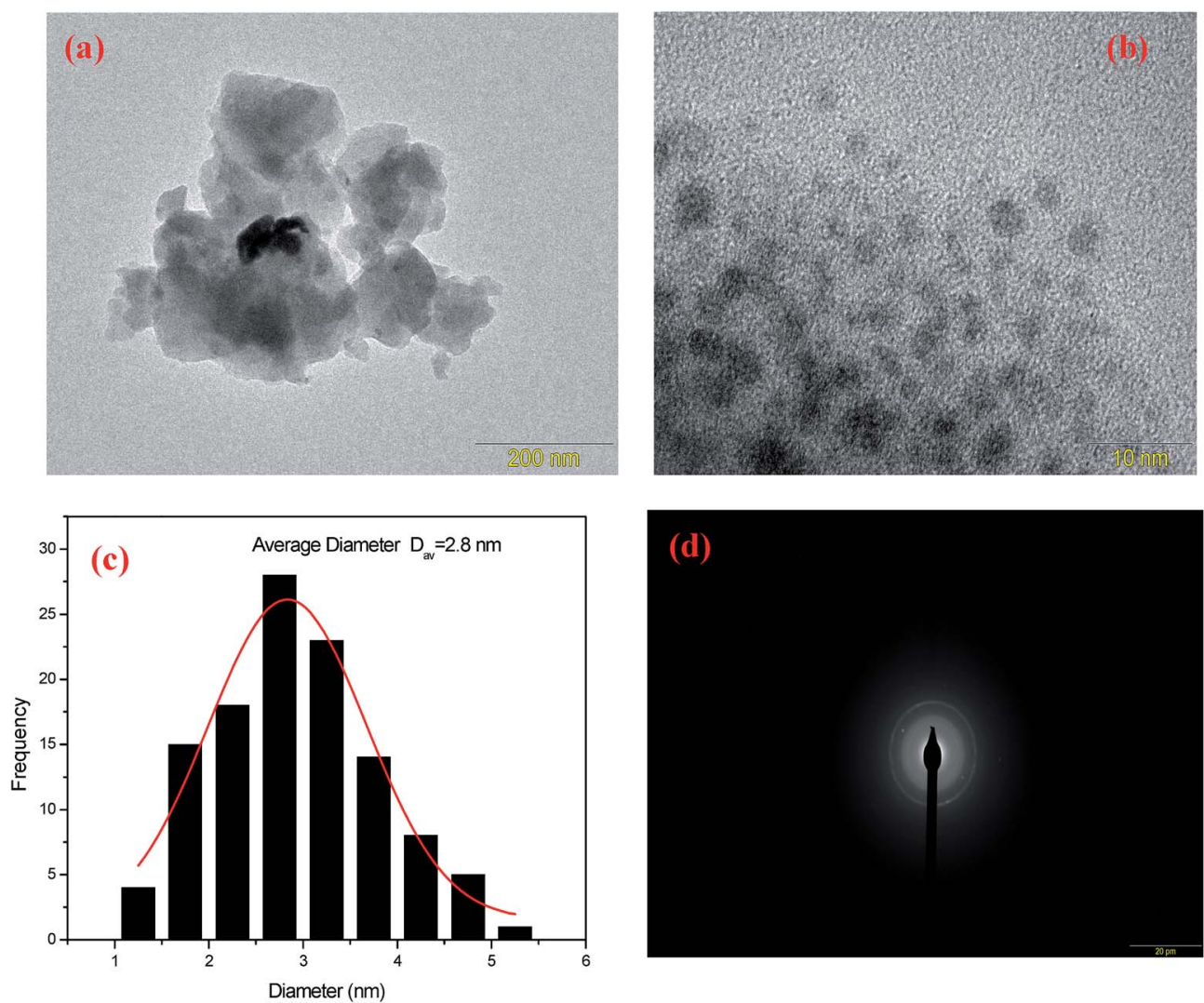

Fig. 4 (a) TEM images (b) at $10 \mathrm{~nm}$ scale, (c) particle size distribution curve and (d) selected area electron diffraction pattern (SAED) of Ag5 glass samples.

the formation of neutral $\mathrm{Ag}^{0}$ particle due to the electrons extracted directly from deffects that are intrinsic to the glass, namely $F^{-}$vacancies residing within fluorophosphate

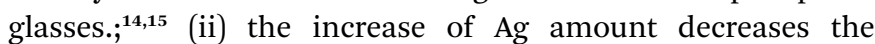
distances among $\mathrm{Ag}^{+}$ions, which interacts to form $\mathrm{Ag}_{2}^{2+} \mathrm{NCs}$. Then, (iii) due to the high mobility of the Ag particles through the fluorophosphate network, the neutral $\mathrm{Ag}$ particle, $\mathrm{Ag}_{2}{ }^{2+} \mathrm{NCs}$ and $\mathrm{Ag}^{+}$particles intrinsically condense, result in the formation of other silver NCs, consisting of a few Ag atoms, defined as $\mathrm{Ag}_{m}{ }^{x+}$ clusters, where $m$ is the number of atoms and $x$ is the formal charge. ${ }^{15}$ When the concentration of $\mathrm{AgNO}_{3}$ is high enough, the reduction rate increases resulting in the enlargement of the size and the concentration of Ag NCs.

\subsection{Photoluminescence dynamics of Ag NCs doped fluorophosphate glasses}

Fig. 5(a) depicts the normalized excitation (PLE) spectra of the basic glass doped with $5 \mathrm{~mol} \%$ of $\mathrm{AgNO}_{3}$. The obtained spectra exhibit broad bands consisting of two individual peaks centered at 270 and $360 \mathrm{~nm}$, respectively. The peak around $270 \mathrm{~nm}$ is related to electronic transition of small $\mathrm{NCs}$ ( $\mathrm{Ag}$ single atom), ${ }^{16,17}$ while we suspect that the broad band centered at $360 \mathrm{~nm}$ and spanning the range from 300 to $410 \mathrm{~nm}$ is related to $\mathrm{Ag}$ NCs comprising different numbers of Ag atoms. ${ }^{16,18}$

The emission spectra of the indicated sample, presented in Fig. 5(b), exhibit a broad luminescence band covering the whole visible range from 350 to $750 \mathrm{~nm}$ when the excitation wavelength is changed from 270 to $400 \mathrm{~nm}$. The position and shape of the emission and excitation spectra match well with the earlier reported respective spectra of Ag NCs dispersed in zeolites, as well as in glass matrix. ${ }^{5,19}$ As it is seen from the pictures in Fig. 5(c), different colors can be generated when the wavelength is changed from $270 \mathrm{~nm}$ to $400 \mathrm{~nm}$. Excitation at wavelengths shorter than $330 \mathrm{~nm}$ results in blueish colors, while excitation at longer wavelengths $(>330 \mathrm{~nm})$ results in warmer (yellowish) whites, due to the higher contributions of the yellow and the red to the total emission spectrum. Furthermore, an evolution in the relative intensity of those bands versus the detection wavelength is observed. Essentially, the band centered at $360 \mathrm{~nm}$ becomes more prominent with increasing the detection wavelength at the expense of the band related to the small NCs at $270 \mathrm{~nm}$. More importantly, the fluorescent profiles revealed a red shift with excitation wavelength. An analogous dependence was observed for the luminescence profile of semiconductor quantum dots (QD's).,20 Tikhomirov et al. explained this behavior by the presence of a variety of Ag NCs with different sizes and geometries, particularly emitting in the blue, green and red region., ${ }^{5,721}$ This is in fact supported by TEM micrographs and histogram for such nanocomposite as shown in Fig. 4(a and c). It can be clearly seen that the $\mathrm{Ag}$ particles have a quite large variation in diameter, $d$, from 1.2 to $5.3 \mathrm{~nm}$. These results are consistent with previous 
(a)

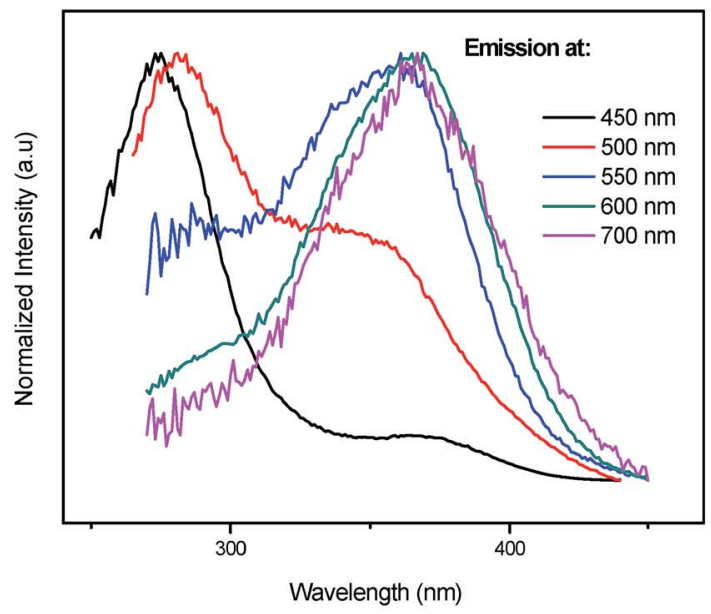

(b)

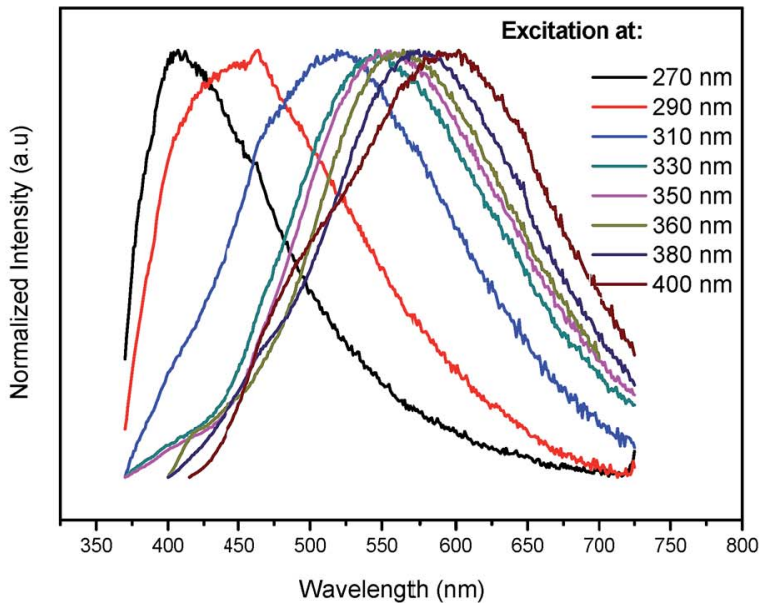

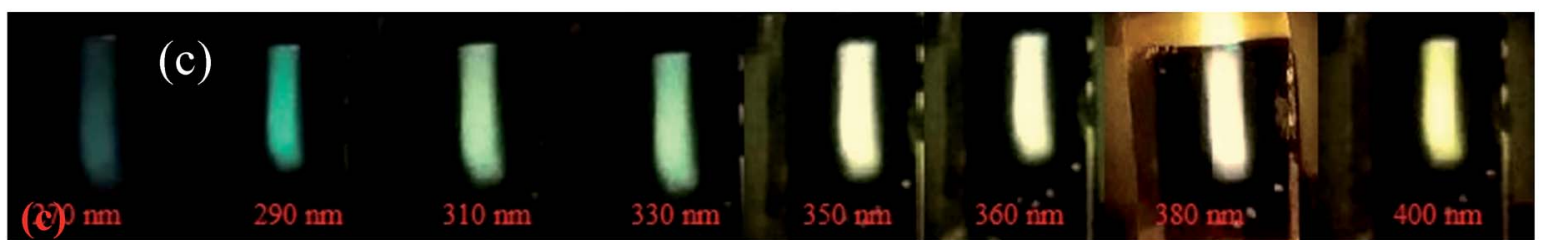

Fig. 5 Normalized (a) excitation and (b) emission spectra of the fluorophosphate glass doped with 5 mol\% $\mathrm{AgNO}_{3}$ monitored/excited at different wavelengths respectively. (c) The corresponding luminescence images showing multicolor emission.

experimental findings for $\mathrm{Ag}$ NCs dispersed in other media. ${ }^{22,23}$ We suspect that isolated $\mathrm{Ag}^{+}$ions, $\mathrm{Ag}_{2}{ }^{2+}$ and $\mathrm{Ag}_{m}{ }^{x+} \mathrm{NCs}$ are responsible for the observed tunable emission band.

Excitation and emission spectra dependences on $\mathrm{Ag}$ NCs concentrations were also investigated. It seems that, the strongest emission occurs in the green-yellow part of the spectrum at $550 \mathrm{~nm}$ and it is most efficient under excitation at $360 \mathrm{~nm}$. On the basis of the above observations, excitation $\left(\lambda_{\mathrm{em}}=550 \mathrm{~nm}\right)$ and emission spectra $\left(\lambda_{\mathrm{ex}}=360 \mathrm{~nm}\right)$ were carried out for different concentrations of $\mathrm{AgNO}_{3}$. The results are shown in Fig. 6 and 7, respectively.

It is clear that these spectra are not affected by the change in oxides/fluorides ratios through varying $\mathrm{AgNO}_{3}$ doping level. In fact, no shifting of the respective excitation and emission spectra of Ag NCs was observed with varying doping level. This invariance indicates that the site of the $\mathrm{Ag}$ NCs does not depend
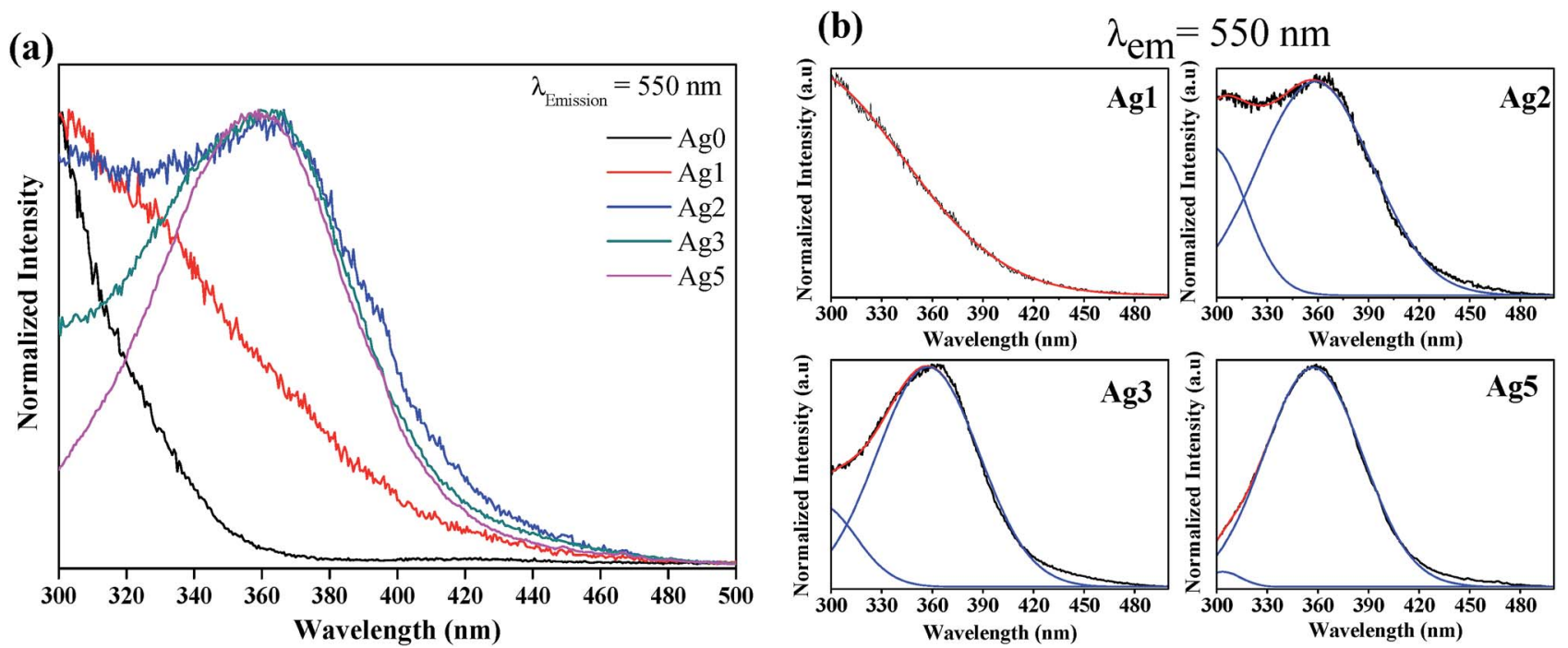

Fig. 6 (a) Normalized and (b) de-convoluted excitation spectra of glass samples as a function of $\mathrm{AgNO}_{3}$ concentration. The emission was fixed at $550 \mathrm{~nm}$. 


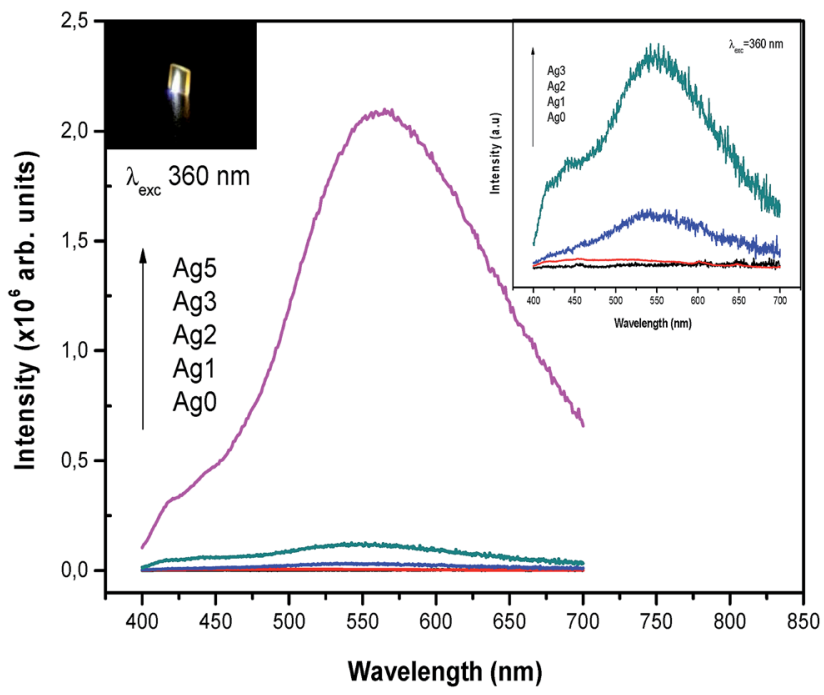

Fig. 7 Emission spectra of prepared samples upon $360 \mathrm{~nm}$ excitation.

on the proportion fluorides/oxide. In contrast, the deconvolution of excitation spectra presented in Fig. 6(b) shows a difference in the relative intensity of the two bands centered at 300 and $360 \mathrm{~nm}$ with the increase of $\mathrm{AgNO}_{3}$ concentration. In fact, the band at higher wavelength (centered at $360 \mathrm{~nm}$ ) is more prominent in Ag5 glass samples at the expense of the band related to the small NCs (centered at $300 \mathrm{~nm}$ ). On the other hand, the emission band related to $\mathrm{Ag}_{m}{ }^{x+} \mathrm{NCs}$ (occurs in the green-yellow part of the spectrum at $550 \mathrm{~nm}$ ), increases drastically with the increase of $\mathrm{AgNO}_{3}$ concentration (Fig. 7). Both results can be explained by an increasing in the silver NCs concentration and/or size with the increase of Ag doping level..$^{16}$ In fact at lower concentration of $\mathrm{AgNO}_{3}$, the glass contains predominantly silver ions $\left(\mathrm{Ag}^{+}\right)$. As the concentration of silver increases, the reduction rate also increases, resulting in increases in the concentration of Ag NCs. Furthermore, the TEM images show that the volume fraction of large NCs increases with the increases of Ag doping level which support our point of view.

\subsection{Heat-treatment dependence of the Ag NCs luminescence}

In order to select the most suitable glass sample with highly luminescence for the target application, we have applied the heattreated technique for different periods, according to the DSC curve of Ag5 sample displayed in Fig. 8. The glass transition $T_{\mathrm{g}}$ and onset crystallization temperature $T_{\mathrm{x}}$ are estimated at $390{ }^{\circ} \mathrm{C}$ and $565{ }^{\circ} \mathrm{C}$, respectively. In such condition, the sample Ag5 was heat-treated above the glass transition temperature $\left(\right.$ at $400{ }^{\circ} \mathrm{C}=T_{\mathrm{g}}+10^{\circ} \mathrm{C}$ ), for different periods $(2,4$, and $6 \mathrm{~h})$, in which the viscosity of glass is reduced for diffusion, aggregation and growth of $\mathrm{Ag}$ NCs. The heattreated samples of Ag5 glass were labeled as R0, R2, R4 and R6, according to the heat-treatment time.

The detailed nanostructure of the $\mathrm{Ag}$ particles within heat treated glasses was analyzed by means of TEM and SAED. The results are shown in Fig. 9. Following this annealing, the TEM micrographs of the sample heat treated for $4 \mathrm{~h}$ (R4) show the

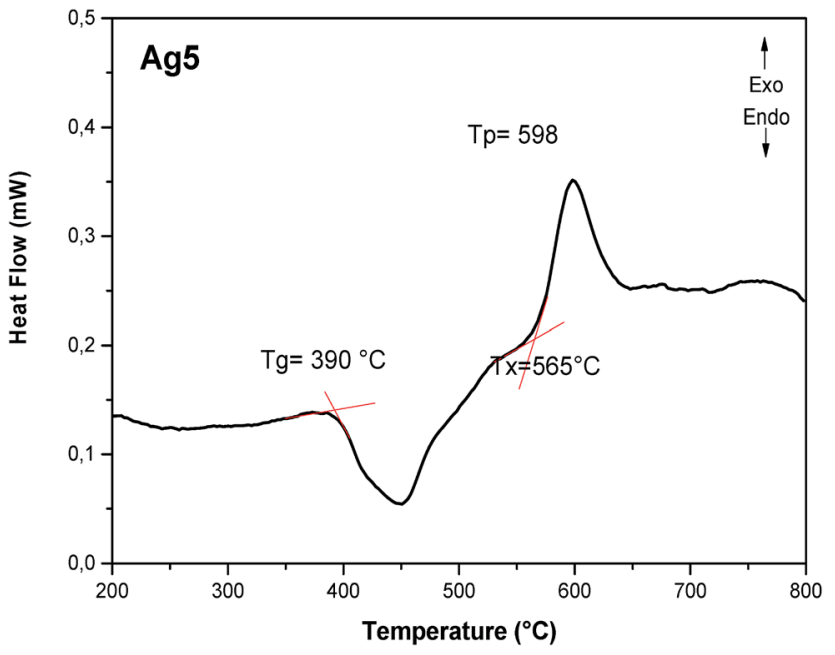

Fig. 8 The DSC curve of Ag5 glass sample.

presence of discrete diffraction rings, striking different from the diffuse halos observed in the as-prepared glass (Fig. 9(a and b)). The quantitative analysis of the size distribution histograms (Fig. 9(c)) reveals that with the heat treatment, the average size of the silver NCs increases from $2.8 \mathrm{~nm}$, for the as-prepared glass (Ag5 denoted in this section R0), to $3.73 \mathrm{~nm}$ after 4 hours of heat treatment. In addition, analysis of high resolution TEM image shows that the spherical particles are consistent with crystal lattice with d-spacing of $0.25 \mathrm{~nm}$, which corresponds to (111) plane of Ag NPs. ${ }^{24}$ Moreover, the selected area electron diffraction (SAED) pattern obtained for the sample annealed for $4 \mathrm{~h}$ also indicates the presence of crystallographic planes, confirming the crystalline character.

The absorption spectra of the corresponding glasses support this point of view (Fig. 10). In fact, the heat-treated glasses show a pronounced plasmon absorption band with a FWHM of $80 \mathrm{~nm}$ in the visible part of the spectrum. This plasmon absorption band increases in intensity and red-shifts with heat treatment time, indicating an increase in the silver NCs size. Taking into account this result, we focus now on the effect of heat treatment on the luminescence properties of silver NCs doped fluorophosphate glasses, with the aim of understanding the relation between the size of $\mathrm{Ag}$ particles, responsible for surface Plasmon effects, and luminescence properties.

The emission spectra of the as-prepared (R0) and heattreated glasses obtained under $360 \mathrm{~nm}$ excitation are shown in Fig. 11. As it can be seen, the emission intensity of Ag NCs doped fluorophosphate glasses decreases after heat treatment. A further heat treatment results in further decrease of the emission, as well as a red shift of the emission band. Combining the TEM results and emission spectra of heattreated glasses, we believe that the quenching of $\mathrm{Ag}$ luminescence with heat treatment is mostly related to the evolution of the amount of $\mathrm{Ag}$ NCs as a function of heat treatment, as indicated by the TEM and emission spectra results. During the heat treatment the luminescent particles aggregates in larger Ag NPs which probably cease to emit as for normal nonluminescent silver metal. ${ }^{14}$ 

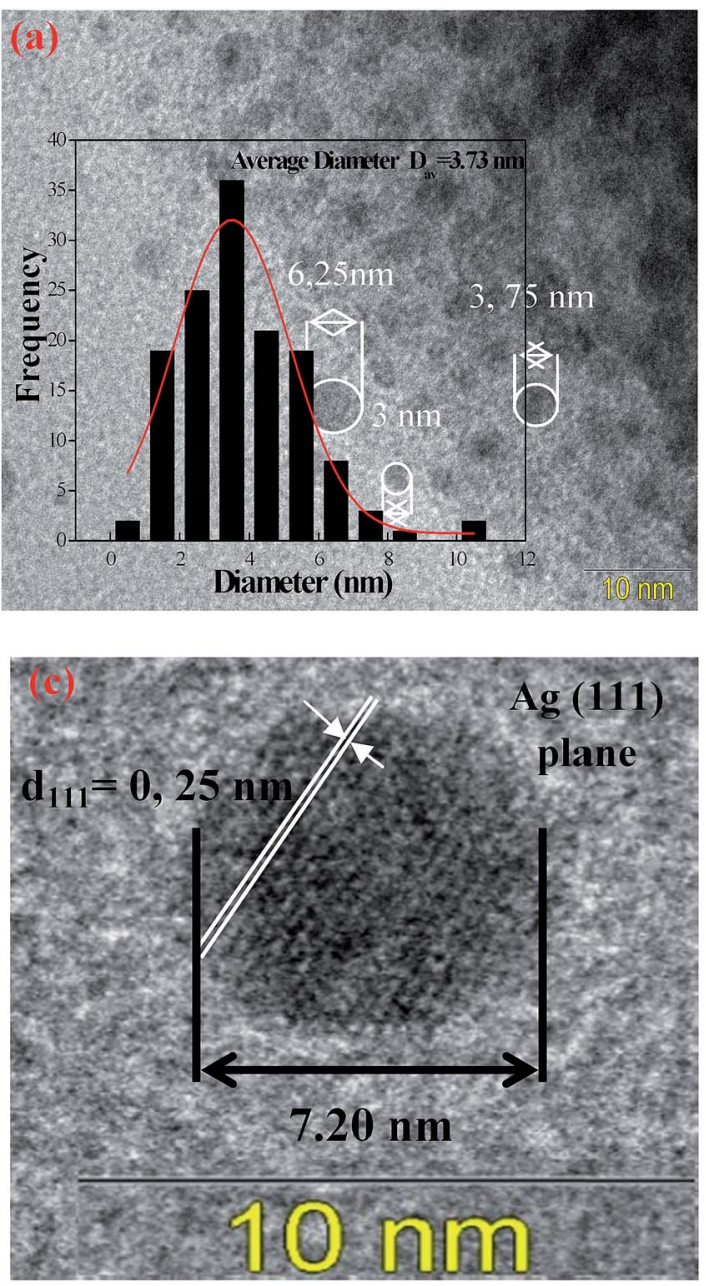
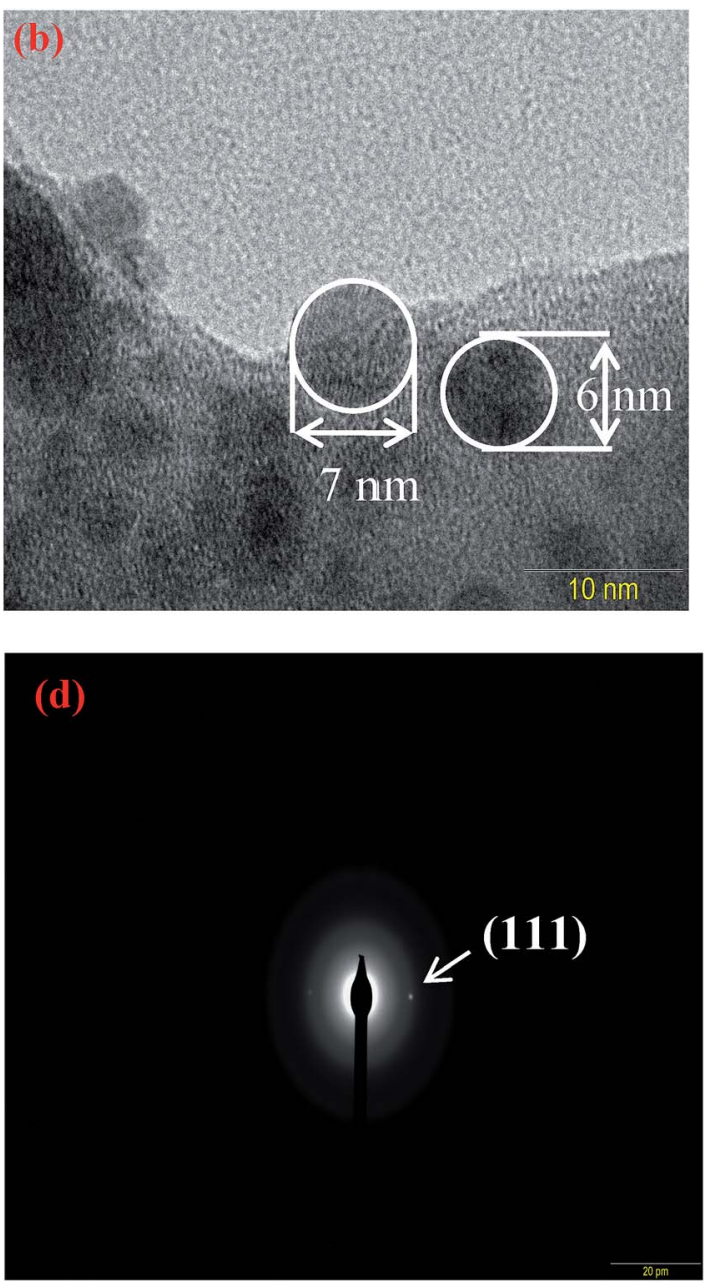

Fig. 9 TEM images, particle size distribution curve and SAED pattern of silver NCs doped fluorophosphate glass after annealing at $400{ }^{\circ} \mathrm{C}$ for $4 \mathrm{~h}$.

In general, plasmonic NPs are known to strongly absorb light, which is related to the collective oscillation of conduction electrons, but they are not luminescent. ${ }^{14}$ In contrast, when the particle size becomes comparable to the Fermi wavelength the

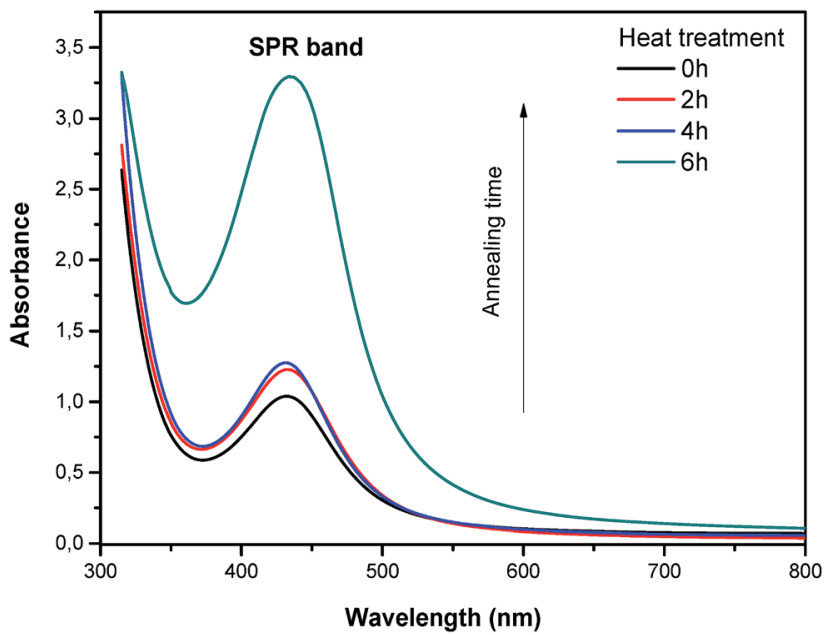

Fig. 10 Absorption spectra of heat treated samples showing pronounced plasmon absorption band. optical properties of $\mathrm{Ag}$ NCs differ from such regime $(\mathrm{Ag}$ nanoparticles). ${ }^{25}$ In this case, metal NCs become molecular species and discrete energy levels arise at the band edges,

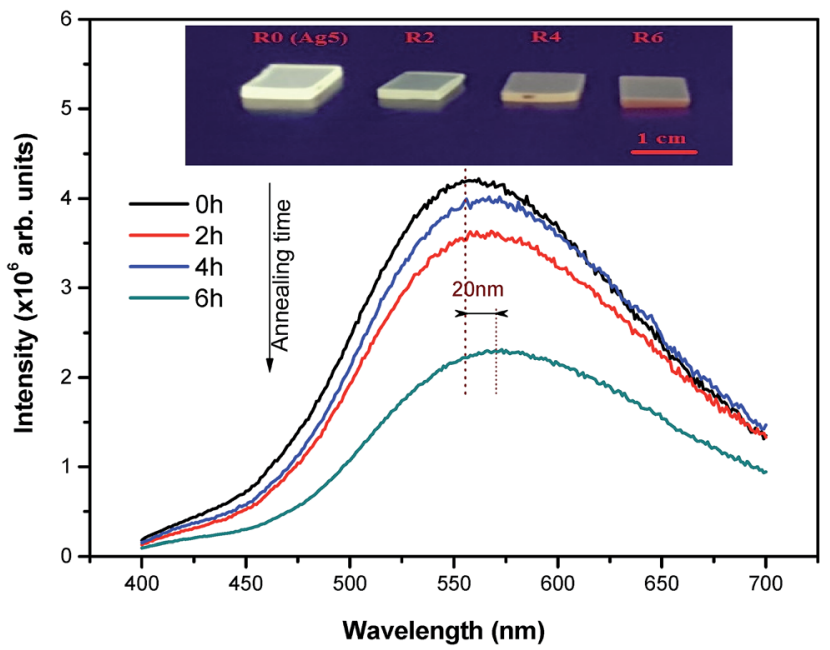

Fig. 11 Emission spectra of the as-prepared and heat treated Ag NCs doped glasses upon $360 \mathrm{~nm}$ excitation. The inset shows pictures of luminescent Ag NCs doped glasses excited by a UV lamp at $365 \mathrm{~nm}$. 


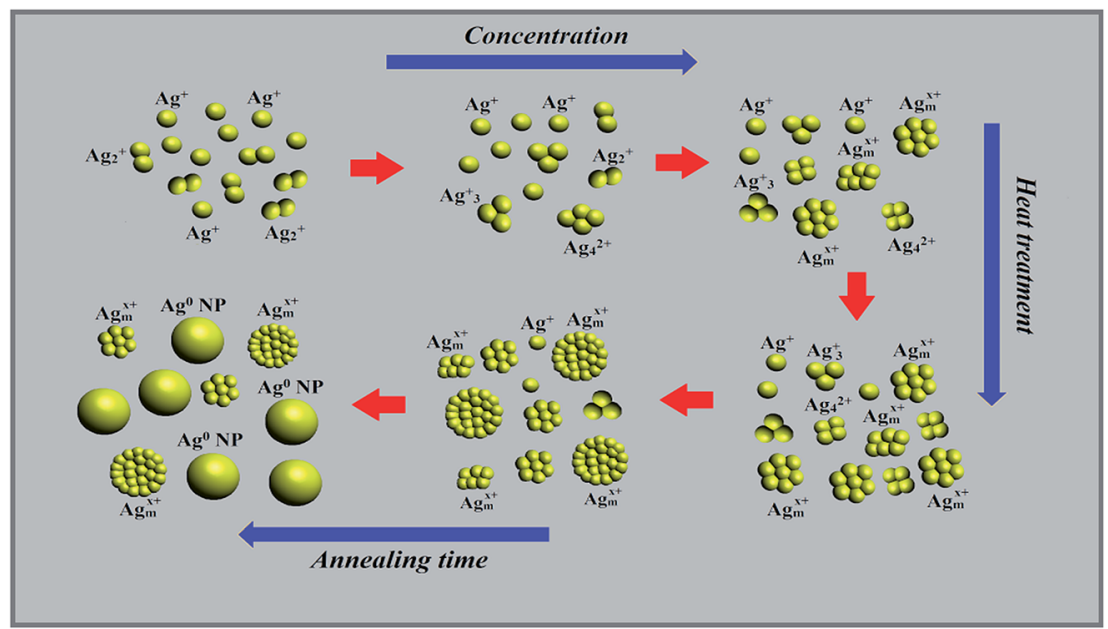

Fig. 12 Schematic illustration of the initial steps of Ag NCs formation, growth and the precipitation of Ag NPs after heat treatment.

making it possible to tune strong fluorescence through a wide spectral window. ${ }^{25}$ These two size-dependent effects are summarized schematically in Fig. 12.

The NCS sizes were varied through silver concentration and thermal annealing. After the first synthetic step, the glass contains predominantly silver ions $\left(\mathrm{Ag}^{+}\right)$and few NCs consisting of a few neutral or charged $\mathrm{Ag}$ particles defined as $\mathrm{Ag}_{m}{ }^{x+} \mathrm{NCs}$. As the concentration of silver increases, the reduction rate also increases, resulting in the enlargement of the size and the concentration of Ag NCs. Presumably, over tens of atoms, Ag NCs aggregates tend to adopt a metallic behavior, evidenced by the SPR in the absorption spectra. From our fluorescence measurements, it was found that the PL intensity, of the samples with low $\mathrm{Ag}$ doping ( $\mathrm{Ag} 1, \mathrm{Ag} 2)$, is weak and increases when further $\mathrm{Ag}$ NCs are formed (Ag3, Ag5) and that different colors can be generated when the excitation wavelength is changed from $270 \mathrm{~nm}$ to $400 \mathrm{~nm}$. This behavior, similar to the luminescence proprieties of quantum dots, suggests a distribution of Ag NCs with different sizes. ${ }^{7,10}$ Thermal treatment above the glass transition temperature leads to the growth of silver NCs in larger Ag NPs. The findings for annealing are the following: (i) the characteristic silver plasmon band evolves in the absorption spectra, (ii) the silver NCs grow into larger NPs, and hence, the number of NCs decreases; (iii) beyond this change in the structure of Ag particles (Ag NCs aggregates into Ag NPs), the PL intensity decreases.

\subsection{Microfabrication and characterization of optical waveguides}

Fig. 13(a) shows an optical microscope image (top view) of the waveguide produced by fs-laser micromachining approximately $200 \mu \mathrm{m}$ below the sample surface. As it can be seen,
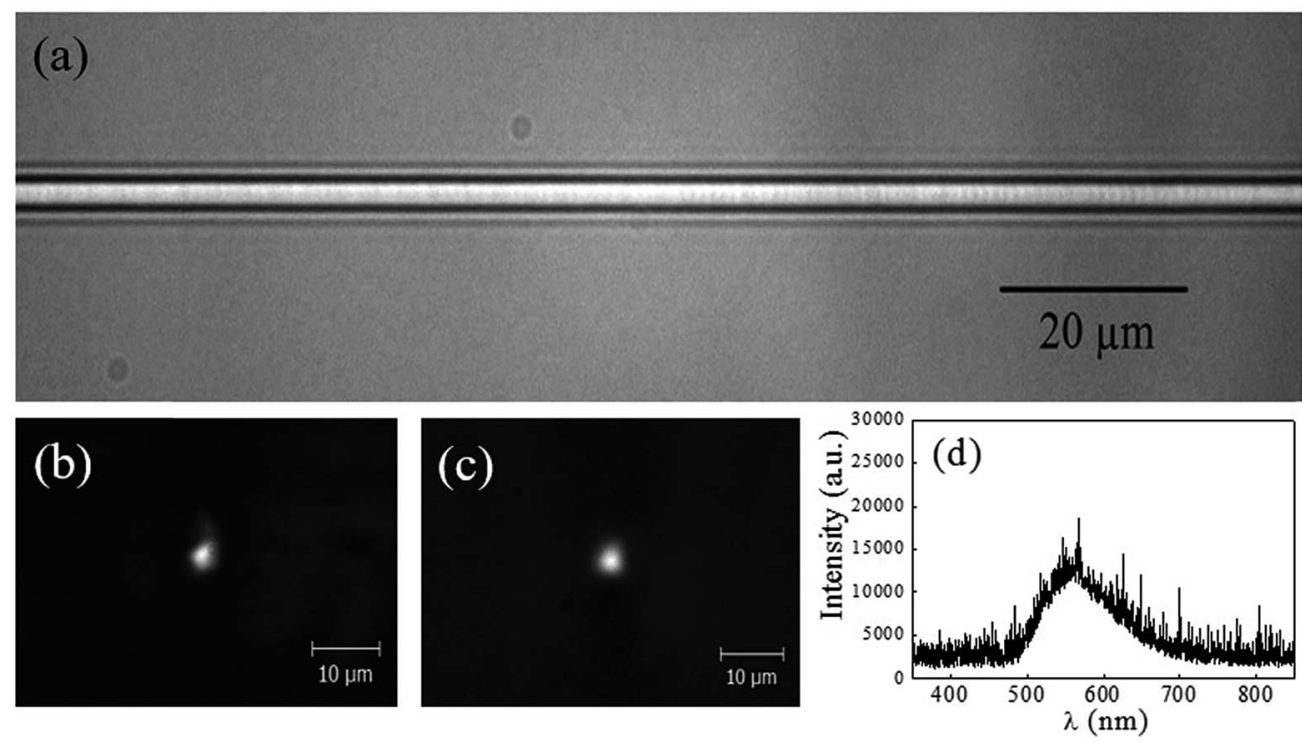

Fig. 13 Images of the waveguide fabricated in the Ag5 sample: (a) top view of waveguide, near-field output profile of the light guided at (b) $632.8 \mathrm{~nm}$ and (c) $325 \mathrm{~nm}$; lastly (d) white light spectrum emitted when coupled with laser $325 \mathrm{~nm}$. 
a continuous and homogeneous waveguide with a diameter of about $5 \mu \mathrm{m}$ was obtained. Fig. 13(b) shows the near-field output profile of the light guided at $632.8 \mathrm{~nm}$, which corresponds to a monomode distribution. Such result indicates a positive change in the refractive index of the material, i.e., the refractive index is larger at the irradiated region than in its surroundness, which allows light confinement. We note that, the difference between the refractive index of the fabricated waveguide and its surrounding is on the order of $2 \times 10^{-3}$. This value was obtained by fitting the intensity profile of the guided mode at $632.8 \mathrm{~nm}$ using a well-known analytical solution. Moreover, the determined total loss, including coupling and propagation losses, is in the order of $15 \mathrm{~dB}$ for the whole length waveguide $(10 \mathrm{~mm})$.

In addition, when laser light at $325 \mathrm{~nm}$ was coupled to the waveguide, it was possible to observe a guided white light in the near-field output profile, as shown in Fig. 13(c). The spectrum of such guided white light is displayed in Fig. 13(d). A redshift in the emission band in comparison with the emission spectrum after laser irradiation is observed (Fig. 5(b)). This behavior can be a consequence of the decrease of $\mathrm{Ag}^{+}$ions concentration in the waveguide core since local heating generated due to the cumulative effect during irradiation might promote aggregation of the reduced $\mathrm{Ag}^{+}$ions into larger Ag NCs. ${ }^{10}$

\section{Conclusion}

In summary, fluorescent silver nanoclusters (Ag NCs) doped fluorophosphate glasses have been prepared using meltquenching method. The shape and the spectral properties were evaluated using transmission electron microscopy (TEM) and photoluminescence measurements. First of all, we show that careful adjustment of the silver concentration and heat treatment lead to glasses with interesting emissive properties that may find several applications such as, for instance, light emitting diodes (LEDs) and other active photonic devices. Lastly, the prepared glass system has been tested as a material for microfabrication of luminescent waveguides. Using the femtosecond laser, homogenous and continuous waveguides were successfully fabricated whose distribution corresponds to a monomode guidance. The main issue for the prepared waveguides is the higher optical loss compared to other waveguides written in other glass system. Considering the fact that the thickness of the waveguide is only $\sim 5 \mu \mathrm{m}$, the results reported herein are still interesting and there is a great potential to reduce the loss by optimizing the concentration of silver NCs and the fabrication conditions including laser spot size, exposure time, energy density and wavelength of laser writing.

\section{Conflicts of interest}

There are no conflicts to declare.

\section{Acknowledgements}

The authors acknowledge Brazilian agency FAPESP (grants numbers \#2016/16343-2, \#2016/11591-8 and \#2013/07793-6) for financial support.

\section{References}

1 M. Yamane and Y. Asahara, Glasses for Photonics, Cambridge University Press, Port Chester, NY, 2000, ISBN 0521580536.

2 G. C. Righini and A. Chiappini, Opt. Eng., 2014, 53, 071819071832.

3 H. Fares, H. Elhouichet, B. Gelloz and M. Férid, J. Appl. Phys., 2014, 116, 123504-123513.

4 H. Fares, W. Stambouli, H. Elhouichet, B. Gelloz and M. Férid, RSC Adv., 2016, 6, 31136-31145.

5 V. K. Tikhomirov, T. Vosch, E. Fron, V. D. Rodríguez, J. J. Velázquez, D. Kirilenko, G. Van Tendeloo, J. Hofkens, M. Van der Auweraer and V. V. Moshchalkov, RSC Adv., 2012, 2, 1496-1501.

6 Y. Ledemi, A.-A. Trudel, V. Rivera, S. Chenu, E. Veron, L. A. O. Nunes, M. Allix and Y. Messaddeq, J. Mater. Chem. C, 2014, 2, 5046-5056.

7 A. S. Kuznetsov, V. K. Tikhomirov, M. V. Shestakov and V. V. Moshchalkov, Nanoscale, 2013, 5, 10065-10075.

8 J. W. Chan, T. R. Huser, S. H. Risbud, J. S. Hayden and D. M. Krol, Appl. Phys. Lett., 2003, 82, 2371-2373.

9 N. Marquestaut, Y. Petit, A. Royon, T. Cardinal and L. Canioni, Adv. Funct. Mater., 2014, 24, 5824-5832.

10 J. M. P. Almeida, P. H. D. Ferreira, D. Manzani, M. Napoli, S. J. L. Ribeiro and C. R. Mendonca, J. Appl. Phys., 2014, 115, 193507-193512.

11 C. B. Schaffer, A. Brodeur and E. Mazur, Meas. Sci. Technol., 2001, 12, 1784-1794.

12 D. M. Krol, J. Non-Cryst. Solids, 2008, 354, 416-424.

13 M. Eichelbaum and K. Rademann, Adv. Funct. Mater., 2009, 19, 2045-2052.

14 V. K. Tikhomirov, V. D. Rodríguez, A. Kuznetsov, D. Kirilenko, G. Van Tendeloo and V. V. Moshchalkov, Opt. Express, 2010, 18, 22032-22040.

15 X. Y. Liu, H. Guo, S. Ye, M. Y. Peng and Q. Y. Zhang, J. Mater. Chem. C, 2015, 3, 5183-5191.

16 H. Fares, T. Castro, J. R. Orives, D. F. Franco and M. Nalin, RSC Adv., 2017, 7, 44356-44365.

17 H. Lin, D. Chen, Y. Yu, R. Zhang and Y. Wang, Appl. Phys. Lett., 2013, 103, 091902-091905.

18 H. X. Xu and K. S. Suslick, ACS Nano, 2010, 4, 3209-3214.

19 G. De Cremer, E. Coutiño-Gonzalez, M. B. J. Roeffaers, B. Moens, J. Ollevier, M. Van der Auweraer, R. Schoonheydt, P. A. Jacobs, F. C. De Schryver, J. Hofkens, D. E. De Vos, B. F. Sels and T. Vosch, J. Am. Chem. Soc., 2009, 131, 3049-3056.

20 I. Díez and R. H. A. Ras, Nanoscale, 2011, 3, 1963-1970.

21 M. V. Shestakov, X. Chen, W. Baekelant, A. S. Kuznetsov, V. K. Tikhomirov, J. Hofkens and V. V. Moshchalkov, RSC Adv., 2014, 4, 20699-20703. 
22 R. Ma, J. Zhao, X. Chen, X. Qiao, X. Fan, J. Du and X. Zhang, Phys. Chem. Chem. Phys., 2017, 19, 22638-22645.

23 A. S. Kuznetsov, V. K. Tikhomirov and V. V. Moshchalkov, Mater. Lett., 2013, 92, 4-6.
24 S. Sareen, V. Mutreja, S. Singh and B. Pal, $R S C A d v .$, 2015, 5, 184-190.

25 P. Kunwar, J. Hassinen, G. Bautista, R. H. A. Ras and J. Toivonen, ACS Nano, 2014, 8, 11165-11171. 